\title{
Free Rides in Logical Space Diagrams versus Aristotelian Diagrams ${ }^{\star}$
}

\author{
Hans Smessaert ${ }^{1[0000-0002-8186-0170]}$, Atsushi \\ Shimojima ${ }^{2[0000-0001-7715-804 X]}$, and Lorenz Demey ${ }^{3[0000-0002-0176-1958]}$ \\ 1 Department of Linguistics, KU Leuven \\ Blijde-Inkomststraat 21, B-3000 Leuven, Belgium \\ https://www.logicalgeometry.org \\ hans.smessaert@kuleuven. be \\ 2 Faculty of Culture and Information Science, Doshisha University, \\ 1-3 Tatara-Miyakodani, Kyotanabe 610-0394, Japan \\ ashimoji@mail.doshisha.ac.jp \\ 3 Center for Logic and Philosophy of Science, KU Leuven \\ Kardinaal Mercierplein 2, B-3000 Leuven, Belgium \\ lorenz. demey@kuleuven. be
}

\begin{abstract}
In this paper we compare two types of diagrams for the representation of logical relations such as contradiction and contrariety, namely Logical Space diagrams (LSD) and Aristotelian diagrams (AD). The cognitive potential of Free Ride - defined in terms of tracking by consequence (Shimojima 2015) - is shown to hold for LSDs but not for ADs. The latter, however, do exhibit a greater inspection potential defined in terms of tracking by correlation. The translational or informational equivalence between LSDs and ADs is contrasted to their lack of computational equivalence and their different degrees of iconicity.
\end{abstract}

Keywords: Cognitive potential · Free Ride - Logical Space diagram . Aristotelian diagram · translation · informational/computational equivalence $\cdot$ degrees of iconicity.

\section{Introduction}

The overall aim of this paper is to apply the general framework for the analysis of diagrams proposed by Shimojima [6] to two different types of diagrams for Aristotelian relations (such as contradiction or contrariety). In Section 1 we briefly present both the general framework - with a special focus on the cognitive potential of Free Ride - and the four Aristotelian relations. In Section 2 we introduce a new representation system for Aristotelian relations, namely LoGICAL Space Diagrams (LSDs), and study their cognitive (Free Ride) potential

\footnotetext{
* The first author acknowledges the financial support from the Research Foundation-Flanders (FWO) of his research stay at Doshisha University with the second author. The third author holds a Postdoctoral Fellowship of the Research Foundation-Flanders (FWO) and a Research Professorship (BOFZAP) from KU Leuven.
} 


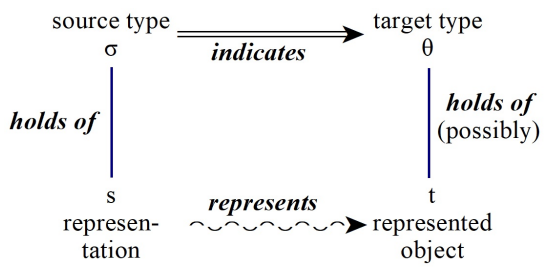

Fig. 1. General framework for the analysis of diagrams [6, Figure 21].

in terms of tracking by consequence. In Section 3 we observe that, although the cognitive potential of Free Ride does not hold of the standard representation of Aristotelian relations by means of ARISTOTELIAN DIAGRAMS (ADs), the latter do allow a weaker mechanism of tracking by correlation. In Sections 4 and 5 we investigate the translation relation between LSDs and ADs, which observes informational equivalence but not computational equivalence, and which reflects differences in degree of iconicity between the two types of diagrams.

General framework for the analysis of diagrams. In order to characterise the semantic content of a diagrammatic representation, the framework adopted in this paper $[6$, p. $23 \mathrm{fff}]$ has a two-tier semantics. It draws a distinction between a TOKEN level at the bottom of Fig. 1 - with a REPRESENTATION relation $\rightsquigarrow$ from a representation $\mathrm{s}$ to represented object $\mathrm{t}$ - and a TYPE level at the top of Fig. 1 - with an INDICATION relation $\Rightarrow$ from a source type $\sigma$ to a target type $\theta$. In the case of a street map, for instance, the representation $\mathrm{s}$ is a particular sheet of paper (token) and the arrangement of lines and symbols is the source type $\sigma$ or property holding of (or 'being suppported' by) that s. The represented object $\mathrm{t}$ is a particular region of a city (token) and the arrangement of streets and buildings is the target type $\theta$ or property holding of that $t$. A representation $\mathrm{s}$ represents an object or situation $\mathrm{t}$ as being of target type $\theta$ if $\mathrm{s}$ represents $\mathrm{t}$ and s supports a source type $\sigma$ that indicates $\theta[6$, p. 27].

Since the notions of consequence tracking and Free Ride will be defined in terms of source and target types, this paper will focus on the type level and the indication relation established by the semantic conventions for the relevant representational practice. ${ }^{4}$ We say that a set $\Gamma$ of source types COLLECTIVELY INDICATES a set $\Delta$ of target types $(\Gamma \Rightarrow \Delta)$ if $\Gamma$ and $\Delta$ stand in a one-to-one correspondence under the indication relation $\Rightarrow$.

Furthermore, a CONSTRAINT is a regularity governing the distribution of sets of types $\Gamma$ and $\Delta$ in a particular class of tokens. There is a constraint $\Gamma \vdash \Delta$ from antecedent set $\Gamma$ to consequent set $\Delta$ if some type in $\Delta$ must hold of a token if all types in $\Gamma$ hold of that token [6, p. 30f]. Thus $\left\{\gamma_{1}, \gamma_{2}\right\} \vdash\left\{\delta_{1}, \delta_{2}\right\}$ means that if $\gamma_{1}$ and $\gamma_{2}$ hold of a token (conjunctively), then $\delta_{1}$ or $\delta_{2}$ must hold of that token (disjunctively). A constraint of the form $\Gamma \vdash\{\delta\}$, with a singleton consequent

\footnotetext{
${ }^{4}$ A semantic convention is essentially arbitrary in its origin, but once people start conforming to it [...] it becomes a "self-perpetuating" constraint over the representational acts of a group of people [6, p. 26].
} 
(a)

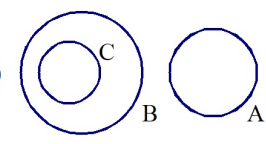

(b) $\Uparrow$

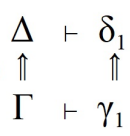

(c)

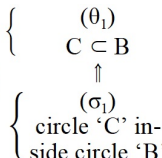

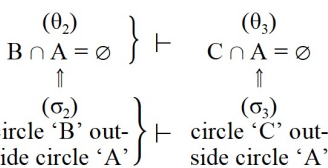

Fig. 2. (a) Free ride in Euler diagram (b-c) Consequence tracking [6, Figure 28-30].

set, is called a CONSEQUENTIAL CONSTRAINT (CC) and rewritten as $\Gamma \vdash \delta$ : if all members of $\Gamma$ hold of a token, then the definite type $\delta$ must hold of it. Constraints may hold both between sets of source types and between sets of target types. It is precisely the correspondences between constraints involving source types and those involving target types that account for many characteristic cognitive potentials of diagrammatic representation systems [6, p. 31].

Free Rides and $\mathbf{C C}$ tracking by consequence. Suppose we take the target types $C \subset B\left(\theta_{1}\right)$ and $B \cap A=\varnothing\left(\theta_{2}\right)$ as the premises of a syllogism at the top of Fig. 2(c). In order for the Euler diagram in Fig. 2(a) to express these two pieces of information, the semantic conventions require us to realise two source types at the bottom of Fig. 2(c), namely the circle labeled ' $\mathrm{C}$ ' is inside the circle labeled ' $\mathrm{B}$ ' $\left(\sigma_{1}\right)$ and the circle labeled ' $\mathrm{B}$ ' is outside the circle labeled ' $\mathrm{A}$ ' $\left(\sigma_{2}\right)$. By virtue of the natural spatial (geometrical and topological) constraints on the arrangements of symbols in Euler diagrams, the realisation of $\sigma_{1}$ and $\sigma_{2}$ automatically realises a third source type, namely that the circle labeled ' $\mathrm{C}$ ' is outside the circle labeled 'A' $\left(\sigma_{3}\right)$. Although this is a side effect of the original operation, $\sigma_{3}$ has an independent semantic value, namely that $C \cap A=\varnothing\left(\theta_{3}\right)$. This target type $\theta_{3}$ is a piece of information that we get 'for free'. Hence, to check the validity of the syllogism, we do not have to infer conclusion $\theta_{3}$ from the premises $\left\{\theta_{1}, \theta_{2}\right\}$. The constraint governing Euler diagrams takes over the work of making the necessary inference, a mechanism called FREE RIDE [6, p. 33]. In the case of a Free Ride potential, expressing a set of information $\Delta$ in a representation automatically results in the expression of other, consequential information $\delta_{1}$. This enables us to skip the mental deductive steps from $\Delta$ to $\delta_{1}$, and to substitute them with the task of reading off $\delta_{1}$ from the representation [6, p. 36]. Fig. 2(b) represents this general constellation of CONSEQUENCE TRACKING. Vertically, $\Gamma$ collectively indicates $\Delta(\Gamma \Rightarrow \Delta)$ and $\gamma_{1}$ indicates $\delta_{1}\left(\gamma_{1} \Rightarrow \delta_{1}\right)$. Horizontally, there is a match ('tracking') between the CC $\Gamma \vdash \gamma_{1}$ on the source types of the representation and the $\mathrm{CC} \Delta \vdash \delta_{1}$ on the target types of the represented object. In order to distinguish it from other types of tracking between source and target type constraints, we refer to the Free Ride mechanism in Fig. 2(b-c) - rendering inference unnecessary - more explicitly as CC TRACKING BY CONSEQUENCE.

Aristotelian relations. In the research programme of Logical Geometry [3, 7] a central object of investigation is the so-called 'Aristotelian square' or 'square of opposition', which visualises logical relations of opposition and implication. 


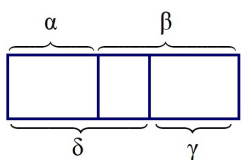

Fig. 3. Logical Space Diagram (LSD).

Table 1 defines these Aristotelian ReLations in an informal way. ${ }^{5}$ Two propositions $\alpha$ and $\beta$ are said to be:

Table 1. Aristotelian relations.
a. contradictory
$C D(\alpha, \beta)$
iff $\alpha$ and $\beta$ cannot be true together and $\alpha$ and $\beta$ cannot be false together
b. contrary
$C(\alpha, \beta)$ iff
$\alpha$ and $\beta$ cannot be true together but
$\alpha$ and $\beta$ can be false together
c. subcontrary
$S C(\alpha, \beta)$ iff $\alpha$ and $\beta$ can be true together but
$\alpha$ and $\beta$ cannot be false together
d. in subalternation $S A(\alpha, \beta)$ iff $\alpha$ entails $\beta$ but $\beta$ doesn't entail $\alpha$

Assuming a meaning postulate relating dead and not alive, the propositions The fly was alive and the fly was dead are contradictory (it has to be one situation or the other, but not both), whereas The fly was alive and The fly was killed are contrary (it may be neither, namely when the fly died a natural death).

\section{CC tracking by consequence in Logical Space diagrams}

Basic syntax and semantics. In Fig. 3 we introduce a new type of diagram, namely the LOgical SPACE Diagram (or LSD for short). The big rectangle represents the complete Logical Space, i.e. the set of possible situations in the world, or the set of all relevant entities of a given logical type (the 'universe of discourse'). Logical Space can then be subdivided in different parts, i.e. subsets of those possible situations, indicated by means of vertical lines inside the big rectangle. ${ }^{6}$ Curly brackets accompanied by a small Greek letter then indicate for which part of Logical Space a given proposition, indicated by the Greek letter, holds, i.e. in which situations that proposition is true, or for which subset of entities of a given logical type a property holds. The LSD format could be considered a notational variant of the Linear Diagram System [1] or the Euler diagram sys$\mathrm{tem}^{7}$ in which an area is visually represented if and only if it is non-empty. One

${ }^{5}$ In model-theoretic semantics, these relations receive a modal definition in terms of the (non-)existence of models/possible worlds in which both formulas are true/false.

${ }^{6}$ Although the full 2D potential of LSD diagrams is not exploited in the present analysis, subdivisions of Logical Space can be both vertical and horizontal.

7 The crucial ingredients are basically the same, namely a universe set $U$ and two subsets $A$ and $B$, which yield four areas to be considered, namely $A \cap B, A \backslash B$, 


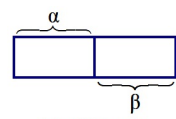

(a) $\operatorname{CD}(\boldsymbol{\alpha}, \boldsymbol{\beta})$

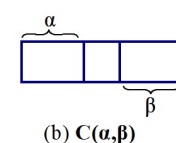

(b) $\mathbf{C}(\boldsymbol{\alpha}, \boldsymbol{\beta})$
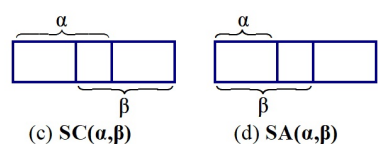

Fig. 4. LSDs for Aristotelian relations.

advantage of such LSDs is the very natural and intuitive representation of the opposition and implication relations.

Aristotelian relations and LSDs. In Fig. 4 we use the Logical Space diagrams to visualise each of the Aristotelian relations defined in Table 1. Three out of the four Aristotelian relations are defined in terms of the two conditions of 'possibly being true together' and 'possibly being false together'. In terms of the LSDs, for two propositions to 'possibly be true together' means that there is an OVERLAP between their two designated areas, whereas for two propositions to 'possibly be false together' means that their is a GAP between their two designated areas. When two propositions are contradictory, the LSD in Fig. 4(a) has no gap and no overlap between their two areas. They are mutually exclusive and jointly exhaustive, and thus yield a perfect bipartition of Logical Space. With the contrary propositions in Fig. 4(b), by contrast, there is no overlap but there is a gap between the two areas. Subcontrary propositions result in the inverse constellation in Fig. 4(c), in which there is no gap but there is an overlap in the middle. Notice that the fourth Aristotelian relation, namely subalternation in Table 1(d), is the odd one out in that it is defined in terms of unidirectional (i.e. asymmetric) entailment instead of in terms of (symmetric) opposition. This hybrid nature of the set of Aristotelian relations in Table 1 is discussed in full detail in [7]. Nevertheless, the LSD for subalternation in Fig. 4(d) can be characterised by means of the same two visual ingredients as the other three relations in Fig. 4(a-c): the two areas designated to the two propositions reveal both a gap (on the right) and an overlap (on the left).

\subsection{CC tracking by consequence with two premises in LSDs}

Remember from the Euler diagram in Fig. 2 above that the starting point for a Free Ride mechanism is the combination of two pieces of information, namely a first relation between objects $\mathrm{A}$ and $\mathrm{B}$, and a second relation between objects $\mathrm{B}$ and C. All four LSDs in Fig. 5 take as their first relation a contradiction between $\alpha$ and $\beta$. The gap in the middle of Fig. $5(\mathrm{a}-\mathrm{b})$ can be characterised either in terms of a contrariety between $\alpha$ and $\gamma$ in Fig. 5(a) or in terms of a subalternation between $\gamma$ and $\beta$ in Fig. 5(b). The overlaps in Fig. 5(c-d) are either due to the subcontrariety between $\beta$ and $\delta$ in the middle of Fig. 5(c) or to the subalternation between $\alpha$ and $\delta$ at the left of Fig. 5(d). Depending on which

$B \backslash A$ and $U \backslash(A \cup B)$ (see also [9]). Given the definition of a proposition as a class of possible worlds, relations between classes in Euler diagrams straightforwardly correspond to relations between propositions in Aristotelian diagrams. 
(a)

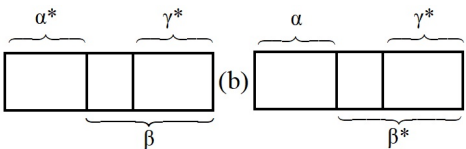

(c)

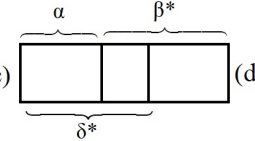

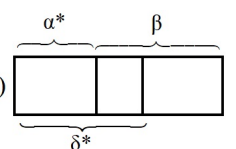

Fig. 5. LSDs for (a-b) $\{\mathrm{C}, \mathrm{CD}, \mathrm{SA}\}$ and (c-d) $\{\mathrm{SC}, \mathrm{CD}, \mathrm{SA}\}$.
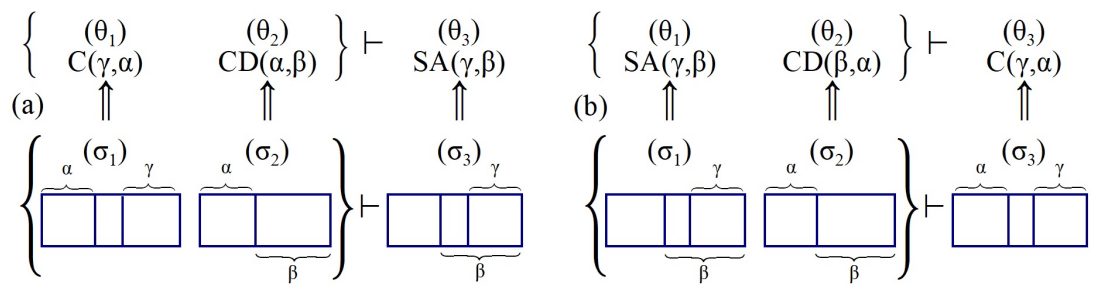

Fig. 6. CC tracking by consequence in LSDs (a) Free Ride 1a: $\{C, C D\} \vdash \mathrm{SA}$ and (b) Free Ride 1b: $\{\mathrm{SA}, \mathrm{CD}\} \vdash \mathrm{C}$.

'perspective' is taken as the second relation - indicated by the asterisks - the four LSDs in Fig. 5 each give rise to their own Free Ride constellation.

Let us first consider the case of Fig. 5(a), spelled out in full detail in Fig. 6(a). The target type $\theta_{1}$ - the contrariety relation $C(\gamma, \alpha)$ - is indicated by the gap in the source type $\sigma_{1} \mathrm{LSD}$, whereas the $\theta_{2}$ contradiction $C D(\alpha, \beta)$ is indicated by the bipartition in the $\sigma_{2}$ LSD. If we now combine $\sigma_{1}$ and $\sigma_{2}$, the natural spatial (geometrical and topological) constraints on the arrangements of symbols in the LSD representation format automatically yield the LSD in Fig. 5(a). The latter now also reveals both a gap and an overlap between $\gamma$ and $\beta$ in $\sigma_{3}$ of Fig. 6(a), and thus conveys a new piece of information 'for free', namely the subalternation relation $S A(\gamma, \beta)$ in $\theta_{3}$. Thus, Fig. 6(a) nicely illustrates the general mechanism of $\mathrm{CC}$ tracking by consequence in Fig. 2(b) above between the $\mathrm{CC}$ on the target level $-\left\{\theta_{1}, \theta_{2}\right\} \vdash \theta_{3}-$ and that on the source level $-\left\{\sigma_{1}, \sigma_{2}\right\} \vdash \sigma_{3}$. The physical operations of drawing a diagram let us project the premises $\left\{\theta_{1}, \theta_{2}\right\}$ of our inference onto an external diagram $\left\{\sigma_{1}, \sigma_{2}\right\}$, exploit the spatial constraints holding there (yielding $\sigma_{3}$ ), and gain a Free Ride to the logical consequence $\theta_{3}$.

Although the LSD is identical in Fig. 5(a) and Fig. 5(b), we get a shift in perspective from the gap between $\alpha$ and $\gamma$ in the former case to the inclusion of $\gamma$ in $\beta$ in the latter case. The corresponding shift from Fig. 6(a) to Fig. 6(b) involves the switch of the first premise $\theta_{1}$ and the conclusion $\theta_{3}$. The two premises $\{S A(\gamma, \beta), C D(\beta, \alpha)\}$ in Fig. 6(b) are expressed by the inclusion of $\gamma$ in $\beta$ in $\sigma_{1}$ and the bipartition between $\alpha$ and $\beta$ in $\sigma_{2}$. By spatial necessity, the combination of the latter two results in the gap between $\alpha$ and $\gamma$ in $\sigma_{3}$, which expresses the valid conclusion of the inference - namely the contrariety relation $C(\gamma, \alpha)$ - as a Free Ride.

Completely analogously, the identical LSDs in Fig. 5(c) and Fig. 5(d) switch the perspective from the overlap between $\beta$ and $\delta$ in the former case to the inclusion of $\alpha$ in $\delta$ in the latter case. The corresponding shift from Fig. 7(a) to 

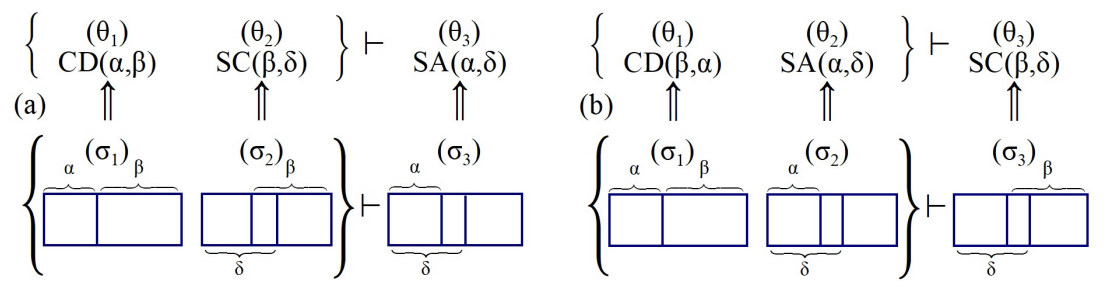

Fig. 7. CC tracking by consequence in LSDs (a) Free Ride 2a: $\{C D, S C\} \vdash$ SA and (b) Free Ride 2b: $\{\mathrm{CD}, \mathrm{SA}\} \vdash \mathrm{SC}$.

(a)

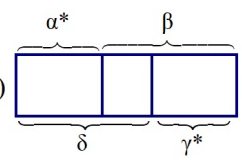

(b)

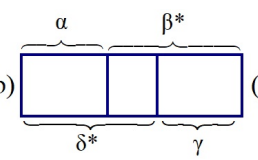

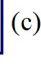

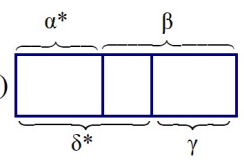

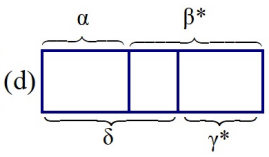

Fig. 8. LSDs for $\{\mathrm{CD}, \mathrm{C}, \mathrm{SC}, \mathrm{SA}\}$.

Fig. 7(b) involves the switch of the second premise $\theta_{2}$ and the conclusion $\theta_{3}$. The two premises $\{C D(\alpha, \beta), S C(\beta, \delta)\}$ in Fig. 7 (a) are indicated by the bipartition between $\alpha$ and $\beta$ in $\sigma_{1}$ and the overlap between $\beta$ and $\delta$ in $\sigma_{2}$. By virtue of the spatial constraints on LSDs, the combination of the latter two necessarily yields the inclusion of $\alpha$ in $\delta$ in $\sigma_{3}$. This in turn serves as a Free Ride and gets interpreted as the valid conclusion of the inference - namely the subalternation $S A(\alpha, \delta)$ in $\theta_{3}$. With the two premises $\{C D(\beta, \alpha), S A(\alpha, \delta)\}$ in Fig. $7(\mathrm{~b})$, the combination of the bipartition between $\alpha$ and $\beta$ in $\sigma_{1}$ and the inclusion of $\alpha$ in $\delta$ in $\sigma_{2}$ automatically results in the overlap between $\beta$ and $\delta$ in $\sigma_{3}$. This Free Ride yields the valid conclusion in $\theta_{3}$ of the subcontrariety relation $S C(\beta, \delta)$.

In both LSD constellations in Fig. 5(a-b) and Fig. 5(c-d) three Aristotelian relations are involved, namely $\{\mathrm{C}, \mathrm{CD}, \mathrm{SA}\}$ and $\{\mathrm{SC}, \mathrm{CD}, \mathrm{SA}\}$ respectively. Both these sets gave rise to two valid syllogisms each, namely Free Ride 1a $\{\mathrm{C}$, $\mathrm{CD}\} \vdash \mathrm{SA}$ in Fig. 6(a), Free Ride $1 \mathrm{~b}\{\mathrm{SA}, \mathrm{CD}\} \vdash \mathrm{C}$ in Fig. 6(b), Free Ride 2a $\{\mathrm{CD}, \mathrm{SC}\} \vdash \mathrm{SA}$ in Fig. $7(\mathrm{a})$ and Free Ride $2 \mathrm{~b}\{\mathrm{CD}, \mathrm{SA}\} \vdash \mathrm{SC}$ in Fig. $7(\mathrm{~b})$. What all these valid patterns have in common is that the CD relation is always one of the premises, whereas the other two relations may serve both as premise and as conclusion. In other words, the third logical combination - with CD in the conclusion and the other two relations as the premises - turns out to be excluded in both cases: $\{\mathrm{C}, \mathrm{SA}\} \nvdash \mathrm{CD}$ and $\{\mathrm{SC}, \mathrm{SA}\} \nvdash \mathrm{CD}$ respectively. What is more, these two pairs of premises are compatible with any possible Aristotelian relation. Although this situation can be related to the notions of OVER-SPECIFICITY and INDETERMINACY in $[6, \mathrm{p} .60 \mathrm{ff}]$, more research is needed to clarify the special status of the CD relation. ${ }^{8}$

\footnotetext{
${ }^{8}$ In particular in terms of the tracking of the two disjunctive constraints $\{\mathrm{C}, \mathrm{SA}\} \vdash$ $\{\mathrm{CD}, \mathrm{C}, \mathrm{SC}, \mathrm{SA}, \mathrm{Un}\}$ and $\{\mathrm{SC}, \mathrm{SA}\} \vdash\{\mathrm{CD}, \mathrm{C}, \mathrm{SC}, \mathrm{SA}, \mathrm{Un}\}$, where Un stands for UNCONNECTEDNESS, the absence of any Aristotelian relation [7].
} 


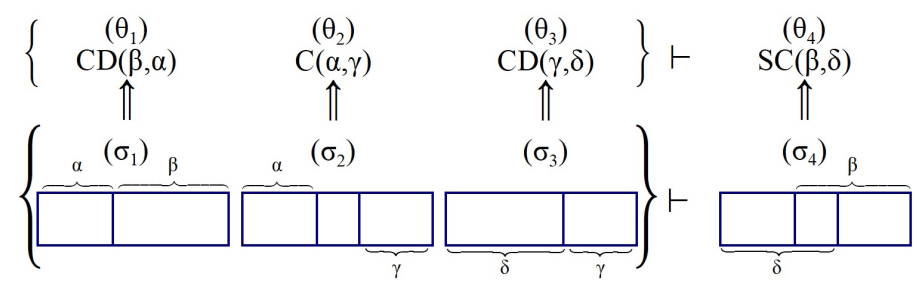

Fig. 9. CC tracking by consequence in LSDs: Free Ride 3a $\{C D, C, C D\} \vdash$ SC.

\subsection{CC tracking by consequence with three premises in LSDs}

Let us now move from CCs with two premises to the constellations in Fig. 8 with three premises. All four LSDs contain two contradiction relations, namely between $\alpha$ and $\beta$ as well as between $\gamma$ and $\delta$. Depending on which 'perspective' is taken as the third relation, these LSDs each give rise to their own Free Ride mechanism.

Taking the gap between $\alpha$ and $\gamma$ in Fig. 8(a) as the second premise in Fig. 9 yields the Free Ride 3a. If you combine the two bipartitions $\alpha-\beta\left(\sigma_{1}\right)$ and $\gamma-\delta$ $\left(\sigma_{3}\right)$ with the $\alpha-\gamma$ gap $\left(\sigma_{2}\right)$, the spatial constraints of LSDs automatically give you the $\beta$ - $\delta$ overlap $\left(\sigma_{4}\right)$, which expresses the conclusion $\theta_{4}$ of the valid syllogism $\{\mathrm{CD}, \mathrm{C}, \mathrm{CD}\} \vdash \mathrm{SC}$. Focussing on the overlap between $\beta$ and $\delta$ in Fig. $8(\mathrm{~b})$, by contrast, would yield a variation of the pattern in Fig. 9 in which the second premise $\sigma_{2} / \theta_{2}$ and the conclusion $\sigma_{4} / \theta_{4}$ are switched around. In other words, whenever you observe a $\beta$ - $\delta$ overlap in combination with the two bipartitions $(\alpha-\beta$ and $\gamma-\delta)$, you get an $\alpha-\gamma$ gap as a Free Ride. This modification of the original Free Ride 3a in Fig. 9 thus results in the Free Ride 3b for the valid syllogism $\{\mathrm{CD}, \mathrm{SC}, \mathrm{CD}\} \vdash \mathrm{C}$. If we take the inclusion of $\alpha$ in $\delta$ in Fig. 8(c) as our perspective, we get the Free Ride $4 \mathrm{a}$ in Fig. 10. The combined observation of the two bipartitions $\alpha-\beta\left(\sigma_{1}\right)$ and $\gamma-\delta\left(\sigma_{3}\right)$ with the gap plus overlap between $\alpha$ and $\delta\left(\sigma_{2}\right)$ by spatial necessity results in the gap plus overlap between $\gamma$ and $\beta\left(\sigma_{4}\right)$, which expresses the conclusion $\theta_{4}$ of the valid syllogism $\{\mathrm{CD}, \mathrm{SA}$, CD $\} \vdash$ SA. And finally, focussing on the inclusion of $\gamma$ in $\beta$ in Fig. 8(d) would yield a variation of the pattern in Fig. 10 in which the second premise $\sigma_{2} / \theta_{2}$ and the conclusion $\sigma_{4} / \theta_{4}$ are again switched around. This modification of the original Free Ride 4a in Fig. 10 thus results in the Free Ride 4b for the same valid syllogism $\{\mathrm{CD}, \mathrm{SA}, \mathrm{CD}\} \vdash \mathrm{SA}$. The observation made at the end of the previous subsection concerning the particular status of the $\mathrm{CD}$ relation turns out to generalise to the patterns with three premises: both in Fig. 9 and Fig. 10 the two CD relations have to be among the premises. Notice, finally, that the combination of Free Rides $4 \mathrm{a}$ and $4 \mathrm{~b}$ in Fig. 8(c-d) and Fig. 10 serves as an elegant visualisation of the Law of Contraposition: $\neg \alpha=\beta$ (CD), $\neg \delta=\gamma(\mathrm{CD})$, $(\alpha \rightarrow \delta)(\mathrm{SA}) \Leftrightarrow(\neg \delta \rightarrow \neg \alpha) \Leftrightarrow(\gamma \rightarrow \beta)(\mathrm{SA})$. 


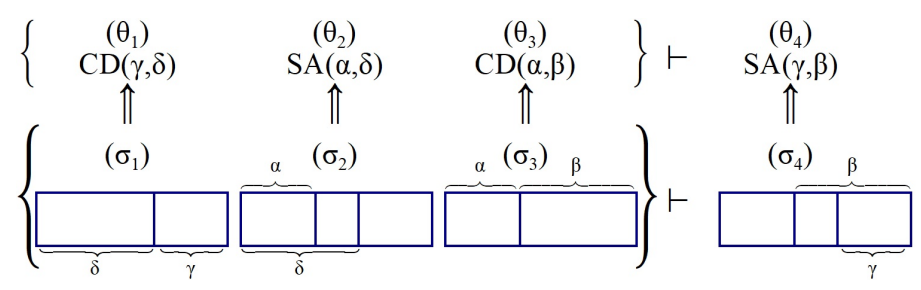

Fig. 10. CC tracking by consequence in LSDs: Free Ride $4 \mathbf{a}\{C D, S A, C D\} \vdash \mathrm{SA}$.

(a)

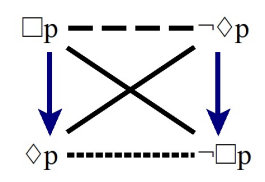

(b)

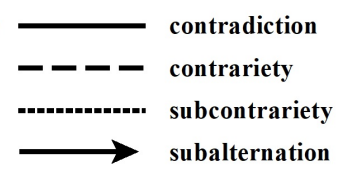

Fig. 11. (a) Aristotelian diagram (AD) and (b) coding conventions.

\section{CC tracking by correlation in Aristotelian diagrams}

Basic syntax and semantics. In order to draw an ARISTOTELIAN DIAGRAM (AD for short), we first of all need a (non-empty) fragment $\mathrm{F}$ of a language $\mathrm{L}$, i.e. a subset of formulas of that language. The formulas in the fragment $\mathrm{F}$ must be contingent and pairwise non-equivalent, and the fragment has to be closed under negation: if formula $\varphi$ belongs to $\mathrm{F}$, then its negation $\neg \varphi$ also belongs to F. For the language S5 of Modal Logic (with operators $\square$ for necessity and $\diamond$ for possibility), for instance, such a fragment $\mathrm{F}$ could be $\{\square p, \neg \square p, \diamond p, \neg \nabla p\}$.

An Aristotelian diagram for $\mathrm{F}$ is then defined as a diagram that visualizes an edge-labeled graph G. Fig. 11(a) presents the AD for the modal fragment $\{\square p$, $\neg \square p, \diamond p, \neg \nabla p\}$. The vertices of $\mathrm{G}$ are the elements of $\mathrm{F}$, whereas the edges of $\mathrm{G}$ are labeled by all the Aristotelian relations holding between those elements, using the coding conventions in Fig. 11(b): full line for CD, dashed line for C, dotted line for SC, and arrow for SA.

Subdiagrams in ADs. From a diagrammatic point of view there are (at least) two ways of looking at a standard square AD. First of all, an AD can be seen as consisting of two triangular subdiagrams: the 'right triangle' in Fig. 12(a) and the 'left triangle' in Fig. 12(b). ${ }^{9}$ Secondly, the AD contains two X-shaped subdiagrams: the 'hour glass' in Fig. 12(c) and the 'bow tie' in Fig. 12(d).

It is important to stress here that these four ARIstotelian SUBDIAGRAMS (henceforth AsDs) are not ADs themselves. Since ADs always consist of an even number of vertices, triangles are excluded in principle. ${ }^{10}$ The hour glass and the bow tie in Fig. 12(c-d), by contrast, do contain an even number of vertices, and do respect the constraint of closure under negation. However, that still does not

\footnotetext{
${ }^{9}$ We ignore the fact that both triangles have a mirror image along the vertical axis.

${ }^{10}$ The right and left triangle in Fig. 12(a-b) are not closed under negation, since the negations of $\gamma$ and $\delta$ are absent from the respective triangles.
} 


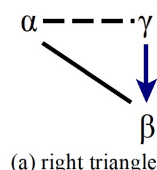

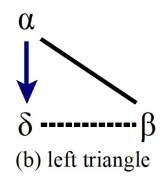
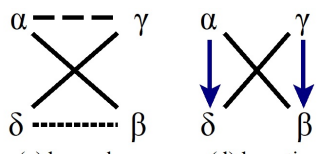

(c) hour glass

(d) bow tie

Fig. 12. Triangular and $\mathrm{X}$-shaped Aristotelian subdiagrams (AsDs).

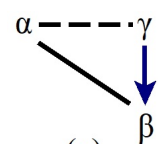

(a)

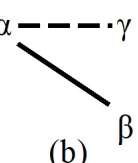

(b)

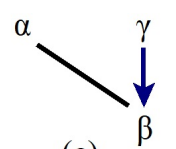

(c)

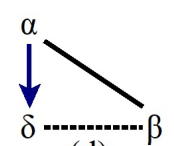

(d)

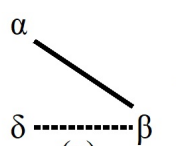

(e)

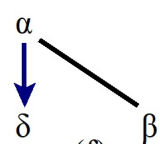

(f)

Fig. 13. AsDs for (a-b-c) $\{C, C D, S A\}$ and (d-e-f) $\{S C, C D, S A\}$.

make them ADs, because the latter have to represent all Aristotelian relations holding between the vertices. ${ }^{11}$ Nevertheless, in spite of the four AsD shapes in Fig. 12 not being ADs themselves, they play a crucial role as the elementary building blocks of such ADs. In the second part of this paper we will precisely demonstrate how these four AsD shapes relate to the different types of Free Ride that were distinguished for the Logical Space diagrams in the first part.

\subsection{CC tracking by correlation with two premises in ADs}

Observe, first of all, that the 'right triangle' in Fig. 12(a) and Fig. 13(a) represents the same $\{\mathrm{C}, \mathrm{CD}, \mathrm{SA}\}$-constellation as the LSDs in Fig. 5(a-b). Hence, Fig. 13(b-c) each represent the two premises of the valid syllogisms $\{C, C D\} \vdash$ SA and $\{\mathrm{SA}, \mathrm{CD}\} \vdash \mathrm{C}$, given in detail in Fig. 14(a) and Fig. 14(b) respectively.

Let us first consider Fig. 14(a) in some detail. Due to the semantic constraints on ADs, we know for sure that - whenever we get from $\gamma$ to $\alpha$ by means of a dashed C-line in $\sigma_{1}^{\prime}$, and from $\alpha$ to $\beta$ by means of a full CD-line in $\sigma_{2}^{\prime}$ - there will be an SA-arrow from $\gamma$ to $\beta$ in $\sigma_{3}^{\prime}$. This is a typical constellation of 'diagram chasing' [2] or 'transitive closure': if you first get from A to B and then from B to $\mathrm{C}$, then you also get from $\mathrm{A}$ to $\mathrm{C}$ directly. On the target type level, Fig. 6(a) and Fig. 14(a) have exactly the same CC (for the valid syllogism). On the source type level of the actual LSD and AD, however, the two are fundamentally different. With the Free Ride 1a in the LSD, there is a matching CC: the $\sigma_{3}$ conclusion from the $\left\{\sigma_{1}, \sigma_{2}\right\}$ premises is a matter of inevitable spatial constraints. Although the $\sigma_{3}^{\prime}$ source type in Fig. 14(a) is equally inevitable, it is not a matter of spatial necessity. There is nothing in the act of drawing the dashed line and the full line for the $\left\{\sigma_{1}^{\prime}, \sigma_{2}^{\prime}\right\}$ 'premises' which would force you to draw the arrow of the $\sigma_{3}^{\prime}$ as a 'conclusion'. Hence, the bottom part of Fig. 14(a) does not constitute a source type level CC, and the overall constellation is not an instance of the Free Ride mechanism.

$\overline{11}$ The hour glass in Fig. 12(c) does not visualise the two vertical SA relations, whereas the bow tie in Fig. 12(d) does not visualise the horizontal $\mathrm{C}$ and $\mathrm{SC}$ relations. 


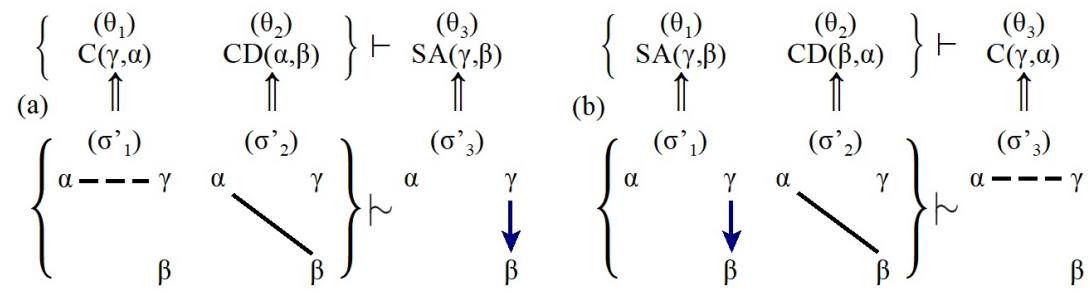

Fig. 14. CC tracking by correlation in $\mathrm{ADs}(\mathrm{a})\{\mathrm{C}, \mathrm{CD}\} \vdash \mathrm{SA}$ and $(\mathrm{b})\{\mathrm{SA}, \mathrm{CD}\} \vdash \mathrm{C}$.

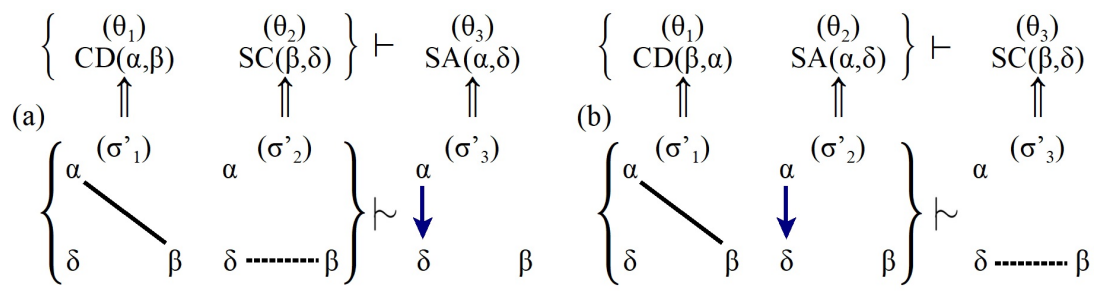

Fig. 15. CC tracking by correlation in $\mathrm{ADs}(\mathrm{a})\{\mathrm{CD}, \mathrm{SC}\} \vdash \mathrm{SA}$ and $(\mathrm{b})\{\mathrm{CD}, \mathrm{SA}\} \vdash \mathrm{SC}$.

Nevertheless, when we inspect a well-formed AD, the combined observation of the two source types $\left\{\sigma_{1}^{\prime}, \sigma_{2}^{\prime}\right\}$ systematically correlates with the observation of $\sigma_{3}^{\prime}$, by virtue of their correspondence (through the indication relation $\Uparrow$ ) with the premises and the conclusion of the $\mathrm{CC}$ on the target type level. In order to reflect this fundamental difference between ADs and LSDs, we replace the turnstile symbol $\vdash$ between the $\left\{\sigma_{1}, \sigma_{2}\right\}$ premises and the $\sigma_{3}$ conclusion in the LSDs with the CORRELATION symbol $\sim$ between the $\left\{\sigma_{1}^{\prime}, \sigma_{2}^{\prime}\right\}$ and the $\sigma_{3}^{\prime}$ source types in the AD. The overall constellation — which is manifestly weaker than that of CC tracking by consequence with the Free Rides in LSDs - will accordingly be referred to as CC TRACKING BY CORRELATION in ADs.

As we observed in connection with the move from Fig. 6(a) to Fig. 6(b), the first premise and the conclusion are switched going from Fig. 14(a) to Fig. 14(b) to yield the second valid inference, i.e. $\{\mathrm{SA}, \mathrm{CD}\} \vdash \mathrm{C}$. Visually speaking, the combined observation of the SA-arrow from $\gamma$ to $\beta$ in $\sigma_{1}^{\prime}$ and the full CD-line from $\beta$ to $\alpha$ in $\sigma_{2}^{\prime}$ by semantic convention correlates with the observation of the dashed C-line from $\gamma$ to $\alpha$ in $\sigma_{3}^{\prime}$. The overall constellation of CC tracking by correlation in Fig. 14(b) thus counts as the weaker counterpart of the Free Ride $1 b$ in Fig. 6(b).

Completely analogously, the 'left triangle' in Fig. 12(b) and Fig. 13(d) represents the same $\{\mathrm{SC}, \mathrm{CD}, \mathrm{SA}\}$-constellation as the LSDs in Fig. 5(c-d). Hence, Fig. 13(e-f) each represent the two premises of the respective valid syllogisms $\{\mathrm{CD}, \mathrm{SC}\} \vdash \mathrm{SA}$ and $\{\mathrm{CD}, \mathrm{SA}\} \vdash \mathrm{SC}$. Thus, the $\mathrm{CC}$ trackings by correlation in Fig. 15(a-b) count as the weaker versions of the $\mathrm{CC}$ trackings by consequence with Free Rides $2 \mathrm{a}$ and $2 \mathrm{~b}$ in Fig. $7(\mathrm{a}-\mathrm{b})$, respectively. As we observed above with the Free Rides in LSDs, in all four valid syllogisms in Fig. 14(a-b) and 
(a)

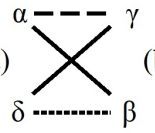

(b)

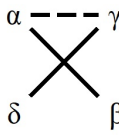

(c)

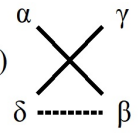

(

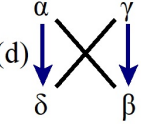

(e)

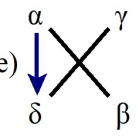

(f)

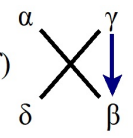

Fig. 16. AsDs for (a-c) $\{\mathrm{CD}, \mathrm{C}, \mathrm{CD}, \mathrm{SC}\}$ and (d-f) $\{\mathrm{CD}, \mathrm{SA}, \mathrm{CD}, \mathrm{SA}\}$.

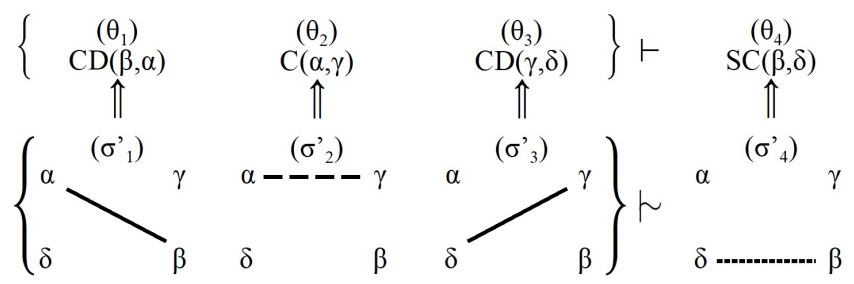

Fig. 17. CC tracking by correlation in ADs: $\{C D, C, C D\} \vdash \mathrm{SC}$.

Fig. 15(a-b) the CD relation is always one of the premises, whereas the other two relations serve both as premise and as conclusion. ${ }^{12}$

\subsection{CC tracking by correlation with three premises in ADs}

At the end of the previous section we moved from the $\mathrm{CC}$ tracking by consequence with two premises to that with three premises. In this subsection we make the corresponding move for CC tracking by correlation. The hour glass pattern in Fig. 12(c) and Fig. 16(a) first of all allows the perspective in Fig. 16(b), which is elaborated in Fig. 17, and which can be seen as the AD counterpart of the LSD Free Ride 3a in Fig. 9. The 'diagram chasing' now consists of three steps from start to finish (instead of two in the previous subsection). By virtue of the semantic constraints on ADs, we know for sure that - whenever we get from $\beta$ to $\alpha$ by means of a full CD-line in $\sigma_{1}^{\prime}$, from $\alpha$ to $\gamma$ by means of a dashed C-line in $\sigma_{2}^{\prime}$, and from $\gamma$ to $\delta$ by means of a full CD-line in $\sigma_{3}^{\prime}$ - there will be a dotted SC-line from $\beta$ to $\delta$ in $\sigma_{4}^{\prime}$. By means of the indication relation, this correlation on the source level is mapped onto the $\mathrm{CC}$ on the target level which captures the valid syllogism $\{\mathrm{CD}, \mathrm{C}, \mathrm{CD}\} \vdash \mathrm{SC}$.

Moving to the second perspective on the hour glass in Fig. 16(c), we get a modification of the configuration in Fig. 17 in which the second 'premise' $\sigma_{2}^{\prime} / \theta_{2}^{\prime}$ and the 'conclusion' $\sigma_{4}^{\prime} / \theta_{4}^{\prime}$ are interchanged. In other words, moving from $\alpha$ to $\beta$ (CD) in $\sigma_{1}^{\prime}$, from $\beta$ to $\delta$ (SC) in $\sigma_{2}^{\prime}$, and from $\delta$ to $\gamma(\mathrm{CD})$ in $\sigma_{3}^{\prime}$ would be equivalent to moving in one big step from $\alpha$ to $\gamma(\mathrm{C})$ in $\sigma_{4}^{\prime}$. This source level correlation is then again mapped onto the target level CC capturing the valid syllogism $\{\mathrm{CD}, \mathrm{SC}, \mathrm{CD}\} \vdash \mathrm{C}$ from the Free Ride $3 \mathrm{~b}$ discussed with Fig. 9.

The two perspectives on the bow tie pattern in Fig. 12(d) and Fig. 16(d), namely Fig. 16(e-f), together yield another visual representation of the Law of

$\overline{12}$ An analysis in terms of OVER-SPECIFICITY or INDETERMINACY [6, p. 60ff] remains a topic for further research. 


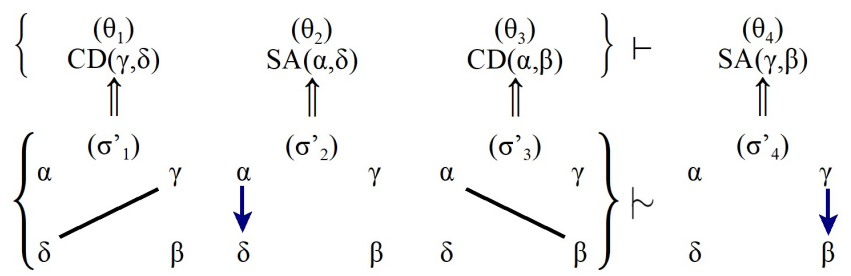

Fig. 18. CC tracking by correlation in ADs: $\{C D, S A, C D\} \vdash \mathrm{SA}$.

(a)

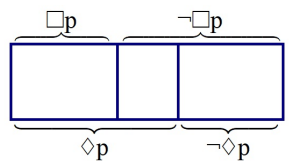

(b)

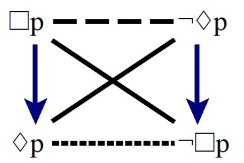

Fig. 19. (a) Logical Space diagram versus (b) Aristotelian diagram.

Contraposition. Fig. 16(e) and Fig. 18 can be seen as the AD counterpart of the LSD Free Ride 4a in Fig. 10. The joint observation of moving from $\gamma$ to $\delta$ (CD) in $\sigma_{1}^{\prime}$, from $\delta$ to $\alpha$ (SA) in $\sigma_{2}^{\prime}$, and from $\alpha$ to $\beta$ (CD) in $\sigma_{3}^{\prime}$ systematically correlates with the observation of the SA move from $\gamma$ to $\beta$. This correlation matches the target level $\mathrm{CC}$ capturing the valid $\{\mathrm{CD}, \mathrm{SA}, \mathrm{CD}\} \vdash \mathrm{SA}$ syllogism. Fig. 16(f) then corresponds to the LSD Free Ride 4b, discussed in connection with in Fig. 10, and requires a modification of Fig. 18 in which $\sigma_{2}^{\prime} / \theta_{2}^{\prime}$ and $\sigma_{4}^{\prime} / \theta_{4}^{\prime}$ are again interchanged. Notice, to conclude, that both in Fig. 17 and Fig. 18, the two CD relations once again have to be among the 'premises' of the correlation.

\section{The translation relation between LSDs and ADs}

From an informational point of view, the Logical Space diagrams introduced in Section 2 and the Aristotelian diagrams introduced in Section 3 are by and large equivalent to one another. The two diagonals for contradiction in Fig. 19(b) correspond to the two bipartitions at the top and the bottom of Fig. 19(a), wheras the two subalternation arrows in Fig. 19(b) correspond to the fact that in Fig. 19(a) the areas for both $\square p$ and $\diamond p$ at the left and those for $\neg \square p$, and $\neg \diamond p$ at the right exhibit both a gap and an overlap. The dashed line for contrariety at the top of Fig. 19(b) reflects the gap between the areas for $\square p$ and $\neg \diamond p$ in Fig. 19(a), while the dotted line for subcontrariety at the bottom of Fig. 19(b) reflects the overlap between the areas for $\neg \square p$ and $\diamond p$ in Fig. 19(a).

In order to capture these systematic correspondences between the two types of representations, we introduce the notion of a translation relation between visual representations. Fig. 20 demonstrates how such a relation fits into an extension of the general framework for the semantic analysis of diagrams introduced in Fig. 1. Remember that the latter's two-tier semantics draws a distinction between the token level and the type level. Hence, at the bottom of Fig. 20, we first 


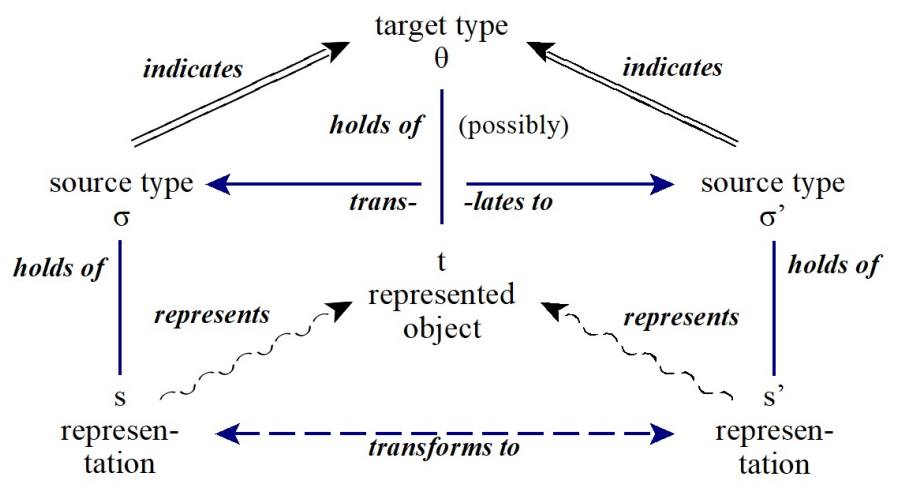

Fig. 20. General framework for the analysis of diagrams in translation.

of all define a TRANSFORMATION relation - indicated with the dashed double arrow - between the two material (token level) representations s (i.e. the LSD) and s' (i.e. the AD). The actual TRANSLATION relation - indicated with the full line double arrow - holds on the type level between the source types $\sigma$ and $\sigma^{\prime}$. The translational equivalence between the latter is then expressed by the fact they both stand in an indication relation with the same target type $\theta$.

The source of the natural language metaphor of translation is straightforward: two natural language expressions (source types $\sigma$ and $\sigma^{\prime}$ ) stand in a relation of translational or informational equivalence with one another if they are mapped onto the same meaning, i.e. the same target type $\theta .{ }^{13}$ Furthermore, the analogy with natural language nicely announces the distinction between informational and computational equivalence [4] to be discussed in the next section. Consider, for instance, the translation relation between a Dutch sentence written in the phonographic Roman alphabet and a Japanese sentence written in kanji and kana signs. Although the sentences may be perfectly equivalent from an informational point of view, there are huge computational or cognitive differences between the two writing system in terms of production, perception and learnability. In the next section, a similar discrepancy will be shown to hold for the two visual representation systems of LSDs and ADs under scrutiny.

\section{Different degrees of iconicity with LSDs and ADs}

The overall constellation of $\mathrm{CC}$ tracking by consequence that gives rise to the Free Ride potential of the LSDs is first and foremost a matter of CONSTRUCTING the diagrams step by step. The weaker constellation of CC tracking by correlation with the ADs, by contrast, is primarily a matter of INSPECTING complete

\footnotetext{
${ }^{13}$ Both with the diagrams in Fig. 20 and with natural languages, we want to emphasize the bidirectionality of the transformation and translation relations, as opposed to the (basic) unidirectionality of the indication and representation relations.
} 
diagrams, i.e. of eye-tracking steps through the diagram. The cognitive utility of the regularities in ADs thus lies in the fact that the transitivity from origin to goal allows you to read off in one big step the effect of two or three small steps. In other words, what you see as 'conclusion' is the logical consequence of what you have read off during the initial inspection of the first two or three steps. Hence, ADs can be considered as convenient pedagogical tools for learning about consequential relationships. Although this inspection value perspective is still there with the LSDs, it is less perspicuous. Since the visual components are more intertwined, more effort is needed, often involving so-called perspective or aspect shifting [6, p. 149ff]. Furthemore, when the structures get more complex - representing Aristotelian relations holding between six or more entities - the greater spatial independence or separation of the relations in the hexagonal (or bigger) ADs results in greater visual clarity and transparency.

The manifest differences between LSDs and ADs in terms of cognitive potentials (or the lack of computational equivalence [4]) can also be related to the semiotic notion of ICONICITY. On the standard view, iconicity is defined in terms of an isomorphism between the structure of the source domain and the structure of the target domain. Hence, the LSDs in this paper would be iconic representations, by virtue of the relationship of similarity between the constellations of surface areas in 2D space and subsets of situations in the outside world. The ADs, by contrast, are - semiotically speaking - no iconic but symbolic representations, without any such relationship of similarity, and purely based on visualisation conventions within a given research community.

On the alternative view proposed in the present paper (see also [5]), however, iconicity is not an object-level relation between structures of objects but rather a meta-level relation of isomorphism between constraints on source types and constraints on target types. ${ }^{14}$ As a consequence, iconicity comes in degrees, depending on the strength of the constraints involved in the isomorphism. The strongest form of iconicity is obviously the one based on CC tracking with Free Rides, as illustrated with the LSDs. Diametrically opposed to the Free Rides with certain diagrammatical representation systems is the total absence of Free Rides in the case of linguistic representation systems [8]. The latter can thus be considered as exhibiting a zero degree of iconicity. The main advantage of this alternative approach to iconicity, however, is that it allows a much more finegrained analysis in terms of various intermediate degrees of iconicity. ${ }^{15}$ Rather than simply dismissing the ADs as symbolic representations, i.e. as non-iconic, they can now be argued to exhibit a weaker, intermediate degree of iconicity. In particular, the overall constellation of $\mathrm{CC}$ tracking by correlation in ADs may be 'weaker' than the CC tracking by consequence with the Free Rides in LSDs,

14 The possible connection with the so-called 'operational' conception of similarity and iconicity in Peirce, as elaborated by Stjernfelt [10, chapter 4] constitutes an intriguing topic of further research.

${ }^{15}$ For example, on this account, Euler diagrams are more iconic than Venn diagrams because they exhibit more constraint trackings. Similarly, the Euler system 2 is stronger and thus more iconic than system 1 since it generates more Free Rides [9]. 
it nevertheless counts as a (partial) isomorphism between sequences of source types and sequences of target types. Finally, also the fact that neither LSDs nor ADs are completely commutative - in the sense that you always need the contradiction relation(s) among the premises - may eventually be accounted for in terms of weaker constraints or intermediate degrees of iconicity.

\section{Conclusion}

In Section 2 we introduced Logical Space Diagrams (LSDs) as a new representation system for Aristotelian relations of opposition and implication, and defined their cognitive (Free Ride) potential in terms of consequential constraint (CC) tracking by consequence. In Section 3 we argued that, although the Free Ride mechanism does not hold of the standard representation by means of ARISTOTELIAN DIAGRAMS (ADs), the latter do allow a weaker mechanism of CC tracking by correlation. In Sections 4 and 5 we investigated the translation relation between LSDs and ADs, which observes informational equivalence but not computational equivalence. CC tracking by consequence with LSDs is crucially a matter of constructing the diagrams step by step, whereas CC tracking by correlation with ADs is primarily a matter of inspecting complete diagrams. This difference relates to differences in degree of iconicity between LSDs and ADs.

\section{References}

1. Bellucci, F., Moktefi, A., Pietarinen, A.V.: Diagrammatic autarchy. Linear diagrams in the 17th and 18th centuries. In: CEUR workshops proceedings 1132, pp. 23-30 (2014)

2. De Toffoli, S.: 'Chasing' the diagram - the use of visualizations in algebraic reasoning. Review of Symbolic Logic 10, 158-186 (2017)

3. Demey, L., Smessaert, H.: Combinatorial bitstring semantics for arbitrary logical fragments. Journal of Philosophical Logic 47, 325-363 (2018)

4. Larkin, J., Simon, H.: Why a diagram is (sometimes) worth ten thousand words. Cognitive Science 11, 65-99 (1987)

5. Shimojima, A.: The graphic-linguistic distinction exploring alternatives. Artificial Intelligence Review 15, 5-27 (2001)

6. Shimojima, A.: Semantic Properties of Diagrams and Their Cognitive Potentials. CSLI Publications (2015)

7. Smessaert, H., Demey, L.: Logical geometries and information in the square of opposition. Journal of Logic, Language and Information 23, 527-565 (2014)

8. Stapleton, G., Jamnik, M., Shimojima, A.: What makes an effective representation of information: A formal account of observational advantages. Journal of Logic, Language and Information 26, 143-177 (2017)

9. Stapleton, G., Shimojima, A., Jamnik, M.: The observational advantages of Euler diagrams with existential import. In: Chapman, P., Stapleton, G., Moktefi, A., Perez-Kriz, S., Bellucci, F. (eds.) Diagrammatic Representation and Inference. pp. 313-329. Springer International Publishing, Cham (2018)

10. Stjernfelt, F.: Diagrammatology: An Investigation On The Borderlines Of Phenomenology, Ontology, And Semiotics. Springer, Dordrecht (2007) 